ISSN 1855-3966 (printed edn.), ISSN 1855-3974 (electronic edn.)

\author{
ARS MATHEMATICA CONTEMPORANEA 16 (2019) 183-202 \\ https://doi.org/10.26493/1855-3974.1378.11d \\ (Also available at http://amc-journal.eu)
}

\title{
Relating the total domination number and the annihilation number of cactus graphs and block graphs*
}

\author{
Csilla Bujtás \\ Faculty of Information Technology, University of Pannonia, \\ Egyetem u. 10, 8200 Veszprém, Hungary \\ Marko Jakovac ${ }^{\dagger}$ \\ Faculty of Natural Sciences and Mathematics, University of Maribor, \\ Koroška cesta 160, 2000 Maribor, Slovenia and \\ Institute of Mathematics, Physics, and Mechanics, \\ Jadranska 19, 1000 Ljubljana, Slovenia
}

Received 10 April 2017, accepted 5 August 2018, published online 22 November 2018

\begin{abstract}
The total domination number $\gamma_{t}(G)$ of a graph $G$ is the order of a smallest set $D \subseteq$ $V(G)$ such that each vertex of $G$ is adjacent to some vertex in $D$. The annihilation number $a(G)$ of $G$ is the largest integer $k$ such that there exist $k$ different vertices in $G$ with degree sum of at most $|E(G)|$. It is conjectured that $\gamma_{t}(G) \leq a(G)+1$ holds for every nontrivial connected graph $G$. The conjecture was proved for graphs with minimum degree at least 3 , and remains unresolved for graphs with minimum degree 1 or 2 . In this paper we establish the conjecture for cactus graphs and block graphs.
\end{abstract}

Keywords: Total domination number, annihilation number, cactus graph, block graph.

Math. Subj. Class.: 05C69

\footnotetext{
* Research of the first author was supported by the National Research, Development and Innovation Office NKFIH under the grant SNN 116095. The second author acknowledges the financial support from the Slovenian Research Agency (research core funding No. P1-0297).

$\dagger$ Corresponding author.

E-mail addresses: bujtas@dcs.uni-pannon.hu (Csilla Bujtás), marko.jakovac@um.si (Marko Jakovac)
} 


\section{Introduction}

All graphs considered in this paper are nontrivial, finite, simple and undirected. By a nontrivial graph we mean a graph on at least two vertices. If $G=(V, E)$ is a graph, then $V=V(G)$ is the set of vertices of order $n(G)=|V|$, and $E=E(G)$ is the set of edges of size $m(G)=|E|$. The degree of a vertex $v \in V$ in graph $G$ will be denoted by $d_{G}(v)$. A vertex $v$ of degree 1 is a leaf, while its only neighbor is called a support vertex. If $u$ has at least two neighbors which are leaves, then $u$ is referred to as a strong support vertex. The minimum and maximum degree among the vertices of $G$ are denoted by $\delta(G)$ and $\Delta(G)$, respectively. For $v \in V(G)$, the set of its neighbors is denoted by $N_{G}(v)$ and called the open neighborhood of $v$. We use a similar notation for a set $A \subseteq V(G)$, it is defined as $N_{G}(A)=\bigcup_{v \in A} N_{G}(v)$. If $G$ is clear from the context, we simply write $d(v), N(v)$ and $N(A)$ instead of $d_{G}(v), N_{G}(v)$ and $N_{G}(A)$, respectively.

For a graph $G$, a set $D \subseteq V(G)$ is a total dominating set if every $v \in V(G)$ has at least one neighbor in $D$; i.e., if $N(D)=V(G)$. If $G$ does not contain isolated vertices, such a set $D$ always exists, and the minimum cardinality of a total dominating set, denoted by $\gamma_{t}(G)$, is the total domination number of $G$. A survey on total domination can be found in [8], and more recently, the topic was thoroughly covered in the book [9]. If $C_{n}$ is a cycle of length $n$, its total domination number can be obtained as follows:

$$
\gamma_{t}\left(C_{n}\right)= \begin{cases}\frac{n}{2}+1, & \text { if } n \equiv 2 \quad(\bmod 4) \\ \left\lceil\frac{n}{2}\right\rceil, & \text { otherwise }\end{cases}
$$

For a set $B \subseteq V$ we denote by $G-B$ the graph which is obtained from $G$ by deleting the vertices in $B$ and all edges incident with them. Moreover, if $v_{1} v_{2} \in E$ and $v_{1} v_{2} \notin E$ with $v_{1}, v_{2} \in V$, we use the notations $G-v_{1} v_{2}$ and $G+v_{1} v_{2}$ for the graphs $\left(V, E \backslash\left\{v_{1} v_{2}\right\}\right)$ and $\left(V, E \cup\left\{v_{1} v_{2}\right\}\right)$, respectively. Let $G_{1}$ and $G_{2}$ be two vertex-disjoint graphs and let $v_{1} \in V\left(G_{1}\right), v_{2} \in V\left(G_{2}\right)$. The identification of vertices $v_{1}$ and $v_{2}$ results in a graph $G$ with $V(G)=\left(V\left(G_{1}\right) \cup V\left(G_{2}\right) \cup\{v\}\right) \backslash\left\{v_{1}, v_{2}\right\}$ such that $N_{G}(v)=N_{G_{1}}\left(v_{1}\right) \cup N_{G_{2}}\left(v_{2}\right)$. Moreover, for any vertex $u \neq v$, the open neighborhood of $u$ remains the same.

The subdivided star $S\left(K_{1, \ell}\right)$ is the graph on $2 \ell+1$ vertices which is constructed from the star $K_{1, \ell}$ by subdividing each edge exactly once (left-hand side of Figure 1). The paw is the graph $P$ obtained from $K_{4}$ by deleting two neighboring edges (right-hand side of Figure 1). A connected graph is called cactus graph if its cycles are pairwise edge-disjoint. Moreover, $G$ is a block graph if each 2-connected component of $G$ is a clique.
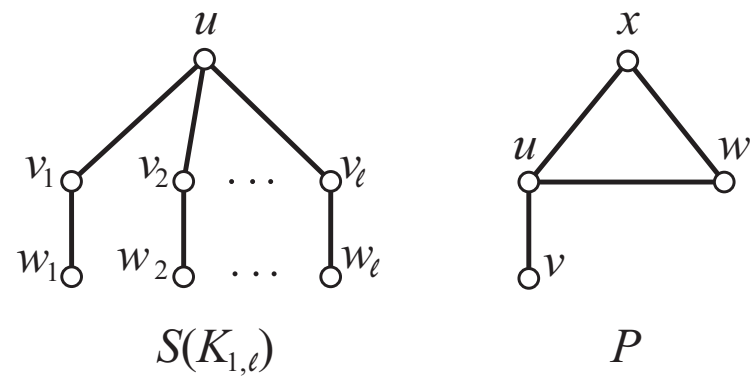

Figure 1: The subdivided star $S\left(K_{1, \ell}\right), \ell \geq 2$, and the paw graph $P$. 
For a subset $S \subseteq V(G)$ we define

$$
\sum(S, G)=\sum_{v \in S} d_{G}(v) .
$$

Let $v_{1}, v_{2}, \ldots, v_{n}$ be an ordering of the vertices of $G$ such that $d\left(v_{1}\right) \leq d\left(v_{2}\right) \leq \cdots \leq$ $d\left(v_{n}\right)$. The annihilation number $a(G)$ is the largest integer $k$ such that $\sum_{i=1}^{k} d\left(v_{i}\right) \leq$ $m(G)$. Equivalently, $a=a(G)$ is the only integer satisfying both

$$
\sum_{i=1}^{a} d\left(v_{i}\right) \leq m(G) \text { and } \sum_{i=1}^{a+1} d\left(v_{i}\right) \geq m(G)+1 .
$$

It is clear by definition that every independent $\operatorname{set}^{1} A$ satisfies $\sum_{v \in A} d(v) \leq m(G)$ and consequently, the annihilation number is an upper bound on the independence number [11]. The annihilation number was first introduced by Pepper in [12]. The 'annihilation process', which is referred to in this original definition, is very similar to the 'Havel-Hakimi process' (see [7] and [11] for exact descriptions).

In general, a set $S$ of vertices is called an annihilation set if $\sum_{v \in S} d(v) \leq m(G)$; and $S$ is an optimal annihilation set, if

$$
|S|=a(G) \quad \text { and } \quad \max \{d(v) \mid v \in S\} \leq \min \{d(u) \mid u \in V(G) \backslash S\} .
$$

In particular, if $G$ is a connected graph on at least 3 vertices, any optimal annihilation set of $G$ contains all leaves.

Assuming that $S$ is an optimal annihilation set, we introduce the following notations. First, denote by $d^{*}(G)$ (or simply by $d^{*}$ ) the minimum vertex degree over the set $V(G) \backslash S$. Note that $d^{*}(G)=d\left(v_{a(G)+1}\right)$, and consequently, the value of $d^{*}(G)$ is independent from the choice of the optimal annihilation set $S$.

The following conjecture can be found in a slightly different form in Graffiti.pc [4], and was later reformulated in [5].

Conjecture 1.1 ([4, 5]). If $G$ is a connected nontrivial graph, then

$$
\gamma_{t}(G) \leq a(G)+1
$$

By definition, every graph satisfies $a(G) \geq\left\lfloor\frac{n(G)}{2}\right\rfloor$. Hence, the formulas given for $\gamma_{t}\left(C_{n}\right)$ above show that each cycle $C_{n}$ satisfies the conjecture. Further, if $\delta(G) \geq 3$, it was observed that the total domination number is at most $\left\lfloor\frac{n(G)}{2}\right\rfloor[1,3,13,14]$. Hence, if $\delta(G) \geq 3$, then $\gamma_{t}(G) \leq a(G)$ clearly holds, even if $G$ is disconnected. Therefore, it is interesting to study this conjecture for graphs with small minimum degree, i.e. $\delta(G) \in$ $\{1,2\}$. So far, Conjecture 1.1 has been proved for only one further important graph class. The following result was established by Desormeaux, Haynes, and Henning in 2013.

Theorem 1.2 ([5]). If $T$ is a nontrivial tree, then $\gamma_{t}(T) \leq a(T)+1$, and the bound is sharp.

\footnotetext{
${ }^{1} \mathrm{~A}$ set $A \subseteq V(G)$ is called an independent set if it induces an edgeless subgraph in $G$. The largest cardinality of such a vertex set is the independence number of $G$ and denoted by $\alpha(G)$.
} 
A similar result was proved by Desormeaux, Henning, Rall, and Yeo [6] for the 2domination number of trees. Very recently, a different proof was given for the same statement by Lyle and Patterson [10]. Namely, their result can be obtained if we replace the total domination number with the 2-domination number in Theorem 1.2.

In this paper we prove Conjecture 1.1 over two further graph classes, namely for cactus graphs and block graphs. These are two natural generalizations of trees and also, for a cactus graph $G$ we have $\delta(G) \leq 2$ and there exist block graphs with small minimum degree. Remark that cactus and block graphs are well-studied classes with several applications, see for instance [2]. Our main results are the following ones.

Theorem 1.3. If $G$ is a nontrivial cactus graph, then $\gamma_{t}(G) \leq a(G)+1$.

Theorem 1.4. If $G$ is a nontrivial block graph, then $\gamma_{t}(G) \leq a(G)+1$.

To formulate and to prove our results we will use the following function $f$ defined for every finite graph $G$ as

$$
f(G)=n(G)+3 m(G)+n_{1}(G)
$$

where $n_{1}(G)$ denotes the number of leaves in $G$. Remark that $f$ is strictly monotone in the sense that if $G^{\prime}$ is a proper subgraph of $G$, then $f\left(G^{\prime}\right)<f(G)$. Indeed, $n\left(G^{\prime}\right)+m\left(G^{\prime}\right)<$ $n(G)+m(G)$ clearly holds, and $2 m\left(G^{\prime}\right)+n_{1}\left(G^{\prime}\right) \leq 2 m(G)+n_{1}(G)$ is true because the deletion of an edge may result in at most two new leaves. Also note that we have $f(G) \geq 7$ for any nontrivial, finite and connected graph $G$.

The paper is organized as follows. In Section 2, we establish several lemmas which will be referred to in later proofs. In Section 3 and 4 we prove Theorem 1.3 and 1.4, respectively. In the last section we discuss the sharpness of our main theorems and arise some related problems.

\section{Preliminary results}

Here we present some preliminary results on how we can obtain a smaller graph $G^{\prime}$ from $G$ (mainly, by deleting some edges and/or vertices from $G$ ) such that $\gamma_{t}\left(G^{\prime}\right) \leq a\left(G^{\prime}\right)+1$ implies $\gamma_{t}(G) \leq a(G)+1$. First we consider changes related to vertices of small degree.

Lemma 2.1. Assume that $G$ is a connected graph on at least three vertices and it fulfills at least one of the following properties:

(i) $d^{*}(G) \leq 2$;

(ii) G has a strong support vertex;

(iii) G contains an induced path v $u_{1} u_{2} u_{3} w$ such that $d\left(u_{1}\right)=d\left(u_{2}\right)=d\left(u_{3}\right)=2$;

(iv) G contains a path $u_{1} u_{2} u_{3} v$ such that $u_{1}$ is a leaf and $d\left(u_{2}\right)=d\left(u_{3}\right)=2$;

(v) G contains two adjacent support vertices.

Then, there exists a nontrivial connected graph $G^{\prime}$ with $f\left(G^{\prime}\right)<f(G)$ such that $\gamma_{t}\left(G^{\prime}\right) \leq$ $a\left(G^{\prime}\right)+1$ implies $\gamma_{t}(G) \leq a(G)+1$. Moreover, if $G$ is a cactus graph, then $G^{\prime}$ can be chosen to be a cactus graph as well; and if $G$ is a block graph, in cases (ii) - (v), $G^{\prime}$ can be chosen to be a block graph. 
Proof. Since trees and cycles satisfy Conjecture 1.1, we may suppose throughout that $G$ is neither a tree nor a cycle.

(i) First assume that $d^{*}(G) \leq 2$. Since $G$ is neither a tree nor a cycle, there exists a vertex $v \in V(G)$ with $d(v) \geq 3$ which is incident to a cycle. Let $e=v u$ be an edge from that cycle. Clearly, $G^{\prime}=G-e$ is connected, $f\left(G^{\prime}\right)<f(G)$ and $m\left(G^{\prime}\right)=m(G)-1$. The deletion of an edge does not decrease the total domination number. This establishes $\gamma_{t}(G) \leq \gamma_{t}\left(G^{\prime}\right)$. Consider now an optimal annihilation set $S^{\prime}$ of $G^{\prime}$. By definition, it satisfies $\sum\left(S^{\prime}, G^{\prime}\right) \leq m\left(G^{\prime}\right)=m(G)-1$. If $u, v \notin S^{\prime}$ then $\sum\left(S^{\prime}, G\right)=\sum\left(S^{\prime}, G^{\prime}\right) \leq$ $m(G)-1$; if $S^{\prime}$ contains exactly one of $u$ and $v$, then $\sum\left(S^{\prime}, G\right)=\sum\left(S^{\prime}, G^{\prime}\right)+1 \leq m(G)$. In either case $a(G) \geq\left|S^{\prime}\right|=a\left(G^{\prime}\right)$ follows. In the third case $u, v \in S^{\prime}$ and $\sum\left(S^{\prime}, G\right)=$ $\sum\left(S^{\prime}, G^{\prime}\right)+2 \leq m(G)+1$. Let $V_{1,2}$ denote the set of vertices which have degree 1 or 2 in $G$. Our assumption $d^{*}(G) \leq 2$ implies $\sum\left(V_{1,2}, G\right) \geq m(G)+1$. Since $d(v) \geq 3$, we have $\sum\left(V_{1,2} \cup\{v\}, G\right) \geq m(G)+4$. Therefore, $\left(V_{1,2} \cup\{v\}\right) \nsubseteq S^{\prime}$ implies that we have a vertex $v^{*} \in V_{1,2}$ which is not contained in $S^{\prime}$. If $v$ is replaced with $v^{*}$ in $S^{\prime}$, we obtain a set $S$ with $\sum(S, G) \leq \sum\left(S^{\prime}, G\right)-1 \leq m(G)$. This proves $a(G) \geq|S|=a\left(G^{\prime}\right)$. If $G^{\prime}$ satisfies (1.1), we may conclude that the same is true for $G$ :

$$
\gamma_{t}(G) \leq \gamma_{t}\left(G^{\prime}\right) \leq a\left(G^{\prime}\right)+1 \leq a(G)+1 .
$$

In the sequel of the proof we will assume that $d^{*}(G) \geq 3$.

(ii) Assume that a vertex $v \in V(G)$ has two neighbors $u_{1}$ and $u_{2}$ which are leaves in $G$. Since $v$ remains a support vertex in $G^{\prime}=G-\left\{u_{1}\right\}$, it is contained in every total dominating set of $G^{\prime}$. This implies $\gamma_{t}\left(G^{\prime}\right)=\gamma_{t}(G)$. On the other hand, every optimal annihilation set of $G$ contains $u_{1}$ and hence $a\left(G^{\prime}\right) \leq a(G)$. Then, $f\left(G^{\prime}\right)<f(G)$, and $\gamma_{t}\left(G^{\prime}\right) \leq a\left(G^{\prime}\right)+1$ implies $\gamma_{t}(G) \leq a(G)+1$.

(iii) If $v u_{1} u_{2} u_{3} w$ is an induced path in $G$ and $d\left(u_{1}\right)=d\left(u_{2}\right)=d\left(u_{3}\right)=2$, consider the graph $G^{\prime}=G-\left\{u_{1}, u_{2}, u_{3}\right\}+v w$. Observe that $n\left(G^{\prime}\right)=n(G)-3, m\left(G^{\prime}\right)=m(G)-3$, $n_{1}\left(G^{\prime}\right)=n_{1}(G)$ and hence, $f\left(G^{\prime}\right)=f(G)-12$. Let $D^{\prime}$ be an optimal total dominating set of $G^{\prime}$ and define $D$ as follows:

$$
D= \begin{cases}D^{\prime} \cup\left\{u_{1}, u_{3}\right\}, & \text { if } v, w \in D^{\prime} ; \\ D^{\prime} \cup\left\{u_{2}, u_{3}\right\}, & \text { if } w \notin D^{\prime} ; \\ D^{\prime} \cup\left\{u_{1}, u_{2}\right\}, & \text { if } w \in D^{\prime} \text { and } v \notin D^{\prime} .\end{cases}
$$

In either case, $D$ is a total dominating set in $G$. Hence, $\gamma_{t}(G) \leq \gamma_{t}\left(G^{\prime}\right)+2$. Consider next an optimal annihilation set $S^{\prime}$ of $G^{\prime}$. Since $d_{G}(v)=d_{G^{\prime}}(v)$ and $d_{G}(w)=d_{G^{\prime}}(w)$, $\sum\left(S^{\prime}, G\right)=\sum\left(S^{\prime}, G^{\prime}\right) \leq m\left(G^{\prime}\right)=m(G)-3$. Our assumption $d^{*}(G) \geq 3$ implies that every vertex $x$ with degree $d(x) \leq 2$ is contained in every optimal annihilation set of $G$. Hence, either $S^{\prime} \cup\left\{u_{1}, u_{2}, u_{3}\right\}$ is a subset of an optimal annihilation set of $G$ and $a(G) \geq a\left(G^{\prime}\right)+3$, or there is a vertex $v^{*} \in S^{\prime}$ with $d\left(v^{*}\right) \geq 3$. In the latter case, consider $S=\left(S^{\prime} \backslash\left\{v^{*}\right\}\right) \cup\left\{u_{1}, u_{2}, u_{3}\right\}$, and observe that $\sum(S, G) \leq \sum\left(S^{\prime}, G\right)-3+3 \cdot 2 \leq m(G)$. Therefore, $a(G) \geq|S|=\left|S^{\prime}\right|+2=a\left(G^{\prime}\right)+2$. If $G^{\prime}$ satisfies inequality (1.1), we have

$$
\gamma_{t}(G) \leq \gamma_{t}\left(G^{\prime}\right)+2 \leq a\left(G^{\prime}\right)+3 \leq a(G)+1
$$

and that proves the statement for property (iii) .

(iv) Let $u_{1} u_{2} u_{3} v$ be a path in $G$ such that $d\left(u_{1}\right)=1$ and $d\left(u_{2}\right)=d\left(u_{3}\right)=2$. Since $G$ is connected and not a path, $G^{\prime}=G-\left\{u_{1}, u_{2}, u_{3}\right\}$ is nontrivial, and we have $f\left(G^{\prime}\right)<f(G)$. 
If $D^{\prime}$ is an optimal total dominating set of $G^{\prime}$, then $D=D^{\prime} \cup\left\{u_{2}, u_{3}\right\}$ totally dominates all vertices in $G$. Thus, $\gamma_{t}(G) \leq|D| \leq \gamma_{t}\left(G^{\prime}\right)+2$. Next, we choose an optimal annihilation set $S^{\prime}$ in $G^{\prime}$ and consider three cases concerning $v$ and $S^{\prime}$.

- If $d(v)=2$, then $G$ contains three consecutive degree-2 vertices and, as we have already proved it in (iii), there exists a graph $G^{\prime}$ with the required property.

- If $v \notin S^{\prime}$, then $\sum\left(S^{\prime}, G\right)=\sum\left(S^{\prime}, G^{\prime}\right)$, and $\sum\left(S^{\prime}, G\right) \leq m\left(G^{\prime}\right)=m(G)-3$. Hence, $S=S^{\prime} \cup\left\{u_{1}, u_{2}\right\}$ satisfies $\sum(S, G)=\sum\left(S^{\prime}, G\right)+3 \leq m(G)$, and $a(G) \geq$ $a\left(G^{\prime}\right)+2$. This, together with the assumption $\gamma_{t}\left(G^{\prime}\right) \leq a\left(G^{\prime}\right)+1$, establishes inequality (1.1) for $G$.

- In the last case we assume that both $v \in S^{\prime}$ and $d(v) \geq 3$ hold. Then, $\sum\left(S^{\prime}, G\right)=$ $\sum\left(S^{\prime}, G^{\prime}\right)+1 \leq m\left(G^{\prime}\right)+1=m(G)-2$. We define $S=\left(S^{\prime} \backslash\{v\}\right) \cup\left\{u_{1}, u_{2}, u_{3}\right\}$ and observe that $\sum(S, G)=\sum\left(S^{\prime}, G\right)-d(v)+5 \leq m(G)$. Hence, $S$ is an annihilation set in $G$ and we may conclude $a(G) \geq|S| \geq a\left(G^{\prime}\right)+2$. The statement of the lemma is proved by the following chain of inequalities: $\gamma_{t}(G) \leq \gamma_{t}\left(G^{\prime}\right)+2 \leq$ $a\left(G^{\prime}\right)+3 \leq a(G)+1$.

(v) Let $u$ and $v$ be two leaves in $G$ with support vertices $u^{\prime}$ and $v^{\prime}$ respectively such that $u u^{\prime}, v v^{\prime}, u^{\prime} v^{\prime} \in E(G)$. Since $G$ is not a path, at least one of these two support vertices, say $u^{\prime}$, is of degree of at least 3. Then, we define $G^{\prime}=G-u u^{\prime}+u v$ and observe that $f\left(G^{\prime}\right)=f(G)-1$. Let $D^{\prime}$ be an optimal total dominating set of $G^{\prime}$. Since $v$ is a support vertex in $G^{\prime}, v \in D^{\prime}$ must hold. Moreover, since $N_{G^{\prime}}(u) \subseteq N_{G^{\prime}}\left(v^{\prime}\right)$, we can choose $D^{\prime}$ such that $u$ does not belong to it. Then, $D=\left(D^{\prime} \backslash\{v\}\right) \cup\left\{u^{\prime}\right\}$ is a total dominating set in $G$. Hence, $\gamma_{t}(G) \leq|D|=\left|D^{\prime}\right|=\gamma_{t}\left(G^{\prime}\right)$. By construction, every vertex has the same degree in $G$ as in $G^{\prime}$ with the two exceptions $v$ and $u^{\prime}$, for which $d_{G}\left(u^{\prime}\right)=d_{G^{\prime}}\left(u^{\prime}\right)+1$ and $d_{G}(v)=d_{G^{\prime}}(v)-1$. Hence, any optimal annihilation set $S^{\prime}$ of $G^{\prime}$ satisfies one of the following cases.

- If $u^{\prime}, v \in S^{\prime}$ or $u^{\prime}, v \notin S^{\prime}$, then $\sum\left(S^{\prime}, G\right)=\sum\left(S^{\prime}, G^{\prime}\right) \leq m\left(G^{\prime}\right)=m(G)$. Therefore, $a(G) \geq\left|S^{\prime}\right|=a\left(G^{\prime}\right)$.

- If $v \in S^{\prime}$ and $u^{\prime} \notin S^{\prime}$, then $\sum\left(S^{\prime}, G\right)=\sum\left(S^{\prime}, G^{\prime}\right)-1 \leq m\left(G^{\prime}\right)-1=m(G)-1$. Therefore, $a(G) \geq\left|S^{\prime}\right|=a\left(G^{\prime}\right)$.

- If $u^{\prime} \in S^{\prime}$ and $v \notin S^{\prime}$, then $\sum\left(S^{\prime}, G\right)=\sum\left(S^{\prime}, G^{\prime}\right)+1 \leq m\left(G^{\prime}\right)+1=m(G)+1$. We define $S=\left(S^{\prime} \backslash\left\{u^{\prime}\right\}\right) \cup\{v\}$. By our assumption, $d_{G}\left(u^{\prime}\right) \geq 3$ and so, we have $\sum(S, G)=\sum\left(S^{\prime}, G\right)-d_{G}\left(u^{\prime}\right)+1 \leq m(G)+1-d_{G}\left(u^{\prime}\right)+1 \leq m(G)-1$. This implies $a(G) \geq a\left(G^{\prime}\right)$.

We have seen that for all possible cases $a\left(G^{\prime}\right) \leq a(G)$ and $\gamma_{t}(G) \leq \gamma_{t}\left(G^{\prime}\right)$. Together with the condition that $G^{\prime}$ satisfies (1.1), these imply $\gamma_{t}(G) \leq \gamma_{t}\left(G^{\prime}\right) \leq a\left(G^{\prime}\right)+1 \leq a(G)+1$.

At the end of the proof we remark that all the above transformations result in a cactus graph $G^{\prime}$, if $G$ was of the same type. Further, with the only exception of $(i)$, the obtained graphs stay block graphs if $G$ is a block graph.

\section{Lemma 2.2.}

(i) For an integer $\ell \geq 3$, let $Q \cong K_{\ell}$ be a complete subgraph of the connected graph $G$ such that $Q$ contains exactly one vertex, say $x$, of degree larger than $\ell-1$. Assume further that $G^{\prime}=G-(V(Q) \backslash\{x\})$ satisfies $\gamma_{t}\left(G^{\prime}\right) \leq a\left(G^{\prime}\right)+1$. Then, $\gamma_{t}(G) \leq$ $a(G)+1$ follows. 
(ii) Let $C$ be a cycle in a connected graph $G$ such that $C$ contains exactly one vertex which is of degree larger than 2. Then, there exists a nontrivial connected graph $G^{\prime}$ with $f\left(G^{\prime}\right)<f(G)$ such that $\gamma_{t}\left(G^{\prime}\right) \leq a\left(G^{\prime}\right)+1$ implies $\gamma_{t}(G) \leq a(G)+1$. Moreover, if $G$ is a cactus graph, then $G^{\prime}$ can be chosen to be a cactus graph as well.

Proof. (i) We suppose $d(x) \geq \ell \geq 3$ and $V(Q)=\left\{v_{1}, v_{2}, \ldots, v_{\ell-1}, x\right\}$. By definition, $m\left(G^{\prime}\right)=m(G)-\left(\begin{array}{l}\ell \\ 2\end{array}\right)$. For any total dominating set $D^{\prime}$ of $G^{\prime}, D^{\prime} \cup\{x\}$ is a total dominating set of $G$. Hence, $\gamma_{t}(G) \leq \gamma_{t}\left(G^{\prime}\right)+1$. Now, let $S^{\prime}$ be an optimal annihilation set in $G^{\prime}$.

- If $x \in S^{\prime}$, we define $S=\left(S^{\prime} \backslash\{x\}\right) \cup\left\{v_{1}, \ldots, v_{\left\lfloor\frac{\ell}{3}\right\rfloor+1}\right\}$. Since $d_{G^{\prime}}(x) \geq 1$, we $\sum(S, G) \leq \sum\left(S^{\prime}, G^{\prime}\right)-1+\left(\left\lfloor\frac{\ell}{3}\right\rfloor+1\right)(\ell-1)$.

- If $x \notin S^{\prime}$, let $S=S^{\prime} \cup\left\{v_{1}, \ldots, v_{\left\lfloor\frac{\ell}{3}\right\rfloor}\right\}$. Then, since $\ell \geq 3$, we have

$$
\sum(S, G) \leq \sum\left(S^{\prime}, G^{\prime}\right)+\left\lfloor\frac{\ell}{3}\right\rfloor(\ell-1) \leq \sum\left(S^{\prime}, G^{\prime}\right)-1+\left(\left\lfloor\frac{\ell}{3}\right\rfloor+1\right)(\ell-1) .
$$

Observe that in either case and for every $\ell \geq 3$, the relation $|S| \geq\left|S^{\prime}\right|+1$ holds. Moreover, as $\sum\left(S^{\prime}, G^{\prime}\right) \leq m\left(G^{\prime}\right)$, we may estimate $\sum(S, G)$ as follows:

$$
\begin{aligned}
\sum(S, G) & \leq m\left(G^{\prime}\right)-1+\left(\left\lfloor\frac{\ell}{3}\right\rfloor+1\right)(\ell-1) \\
& =m(G)-\left(\begin{array}{l}
\ell \\
2
\end{array}\right)-1+\left(\left\lfloor\frac{\ell}{3}\right\rfloor+1\right)(\ell-1) \\
& =m(G)-\left[(\ell-1)\left(\frac{\ell}{2}-\left\lfloor\frac{\ell}{3}\right\rfloor-1\right)+1\right] \leq m(G) .
\end{aligned}
$$

Here, the last inequality can be directly checked for $\ell=3,4$ and 5 . If $\ell \geq 6$, this clearly follows from $\frac{\ell}{2}-\frac{\ell}{3}-1 \geq 0$. We conclude that $S$ is an annihilation set in $G$ and therefore, $a(G) \geq|S| \geq\left|S^{\prime}\right|+1=a\left(G^{\prime}\right)+1$. Together with the condition given in $(i)$ for $G^{\prime}$,

$$
\gamma_{t}(G) \leq \gamma_{t}\left(G^{\prime}\right)+1 \leq a\left(G^{\prime}\right)+2 \leq a(G)+1
$$

follows. This finishes the proof of $(i)$.

(ii) Since $C_{3}=K_{3}$, it suffices to prove (ii) for cycles $C \cong C_{\ell}$ of length $\ell \geq 4$. If $d^{*}(G) \leq 2$ or $\ell \geq 6$, Lemma 2.1(i) and 2.1(iii) establish the statement. Henceforth, we will suppose that $d^{*}(G) \geq 3$ and $\ell=4$ or 5 . Let $x v_{1} \ldots v_{\ell-1} x$ be the cycle $C$ such that $d(x) \geq 3$.

First, assume that $\ell+d_{G}(x) \geq 8$; i.e., at least one of $\ell=5$ and $d_{G}(x) \geq 4$ holds. Let $G^{\prime}=G-(V(C) \backslash\{x\})$ and let $D^{\prime}$ be an optimal total dominating set of $G^{\prime}$. Observe that $D=D^{\prime} \cup\left\{v_{2}, v_{3}\right\}$ is a total dominating set in $G$ and consequently, $\gamma_{t}(G) \leq \gamma_{t}\left(G^{\prime}\right)+2$. Now, fix an optimal annihilation set $S^{\prime}$ in $G^{\prime}$ and consider the following two subcases.

- If $x \notin S^{\prime}$, we have $\sum\left(S^{\prime}, G\right)=\sum\left(S^{\prime}, G^{\prime}\right) \leq m\left(G^{\prime}\right)=m(G)-\ell$. Then, we define $S=S^{\prime} \cup\left\{v_{1}, v_{2}\right\}$ and observe that $\sum(S, G)=\sum\left(S^{\prime}, G\right)+2 \cdot 2 \leq m(G)-\ell+4 \leq$ $m(G)$. This proves $a(G) \geq|S|=a\left(G^{\prime}\right)+2$.

- If $x \in S^{\prime}$, we have $\sum\left(S^{\prime}, G\right)=\sum\left(S^{\prime}, G^{\prime}\right)+2 \leq m\left(G^{\prime}\right)+2=m(G)-\ell+2$. In this case, consider $S=\left(S^{\prime} \backslash\{x\}\right) \cup\left\{v_{1}, v_{2}, v_{3}\right\}$. For this set,

$$
\sum(S, G) \leq \sum\left(S^{\prime}, G\right)-d_{G}(x)+3 \cdot 2 \leq m(G)-\ell-d_{G}(x)+8 \leq m(G)
$$


holds under the present assumption $\ell+d_{G}(x) \geq 8$. Therefore, we have $a(G) \geq$ $|S|=a\left(G^{\prime}\right)+2$.

In either subcase, if $G^{\prime}$ satisfies (1.1), we may conclude that

$$
\gamma_{t}(G) \leq \gamma_{t}\left(G^{\prime}\right)+2 \leq a\left(G^{\prime}\right)+3 \leq a(G)+1
$$

In the other case, $C \cong C_{4}$ and $d_{G}(x)=3$. Here, we define $G^{\prime}=G-V(C)$. Since $d_{G}(x)=3, G^{\prime}$ is connected. If $G^{\prime}$ consists of only one vertex, $\gamma_{t}(G)=2<a(G)+1$ can be proved directly. Hence, we may assume that $G^{\prime}$ is nontrivial. Let $D^{\prime}$ be an optimal total dominating set in $G^{\prime}$ and observe that, also in this case, $D=D^{\prime} \cup\left\{v_{2}, v_{3}\right\}$ is a total dominating set in $G$. Hence, $\gamma_{t}(G) \leq \gamma_{t}\left(G^{\prime}\right)+2$. On the other hand, let $S^{\prime}$ be an optimal annihilation set in $G^{\prime}$. Since there is at most one edge between $S^{\prime}$ and $V(C)$, $\sum\left(S^{\prime}, G\right) \leq \sum\left(S^{\prime}, G^{\prime}\right)+1 \leq m\left(G^{\prime}\right)+1=m(G)-4$. Moreover, for $S=S^{\prime} \cup\left\{v_{1}, v_{2}\right\}$, we obtain $\sum(S, G)=\sum\left(S^{\prime}, G\right)+4 \leq m(G)$, from which $a(G) \geq a\left(G^{\prime}\right)+2$ follows. Thus, if $G^{\prime}$ satisfies (1.1), the desired inequality $\gamma_{t}(G) \leq a(G)+1$ holds again.

The analogue of the following proof was given by Desormeaux et al. [5] inside the proof of Theorem 1.2. There, both $H$ and $T$ were restricted to be a tree. Here, we restate and prove the lemma in a more general form, where $H$ can be an arbitrary connected graph.

Lemma 2.3. Let $H$ be a nontrivial connected graph and $T$ be a tree such that $V(H) \cap$ $V(T)=\emptyset$. Suppose that $w \in V(H), u \in V(T)$, and $v$ is a leaf in $T$ such that $d(u, v) \geq 3$. If $G$ is obtained from $H$ and $T$ by identifying $w$ and $u$, there exists a connected graph $G^{\prime}$ with $f\left(G^{\prime}\right)<f(G)$ such that $\gamma_{t}\left(G^{\prime}\right) \leq a\left(G^{\prime}\right)+1$ implies $\gamma_{t}(G) \leq a(G)+1$.

Proof. First note that the statement follows from Lemma 2.1(i) if $d^{*}(G) \leq 2$. Hence, we may suppose that $d^{*}(G) \geq 3$. Assume that $T$ is rooted in $u$ and choose a leaf $v_{1} \in V(T)$ which is of maximum distance from $u$. Let $v_{2}$ be the parent of $v_{1}$, and $v_{3}$ be the parent of $v_{2}$. By assumption, $d\left(u, v_{1}\right) \geq 3$ and hence, $v_{i} \neq u(i=1,2,3)$.

We will consider graphs $G^{\prime}$ obtained from $G$ by removing a set of vertices from $V(T)$ in such a way that $G^{\prime}$ will stay connected. Throughout, $S^{\prime}$ will denote an optimal annihilation set in $G^{\prime}$.

If $v_{2}$ is a strong support vertex, Lemma 2.1(ii) implies the statement. So, we may suppose that $v_{1}$ is the only leaf of the support vertex $v_{2}$. Since $v_{1}$ is of maximum distance from $u, d\left(v_{2}\right)=2$ also follows. Remark that the same is true for any other leaf and its support vertex, if the leaf is of maximum distance from $u$. Suppose that $d\left(v_{3}\right) \geq 3$ and let $G^{\prime}=G-\left\{v_{1}, v_{2}\right\}$. So $m\left(G^{\prime}\right)=m(G)-2$. If $v_{3}$ is a support vertex in $G^{\prime}$, then $v_{3}$ belongs to a minimum total dominating set $D^{\prime}$ of $G^{\prime}$. If $v_{3}$ is not a support vertex, then every child of $v_{3}$ is a support vertex of degree 2 . If a leaf-neighbor of a child of $v_{3}$ belongs to $D^{\prime}$, then we can simply replace it in $D^{\prime}$ with the vertex $v_{3}$. In either case, we may assume that $v_{3} \in D^{\prime}$. Thus the set $D=D^{\prime} \cup\left\{v_{2}\right\}$ is a total dominating set of $G$, and so $\gamma_{t}(G) \leq|D|=\left|D^{\prime}\right|+1=\gamma_{t}\left(G^{\prime}\right)+1$. Independently of whether vertex $v_{3}$ lies in $S^{\prime}$ or not we have $\sum\left(S^{\prime}, G\right) \leq \sum\left(S^{\prime}, G^{\prime}\right)+1 \leq m\left(G^{\prime}\right)+1=m(G)-1$. Consider $S=S^{\prime} \cup\left\{v_{1}\right\}$. Then $\sum(S, G)=\sum\left(S^{\prime}, G\right)+\bar{d}\left(v_{1}\right) \leq m(G)$, implying that $a(G) \geq|S|=\left|S^{\prime}\right|+1=a\left(G^{\prime}\right)+1$. By assumption, we have that $\gamma_{t}\left(G^{\prime}\right) \leq a\left(G^{\prime}\right)+1$. Therefore,

$$
\gamma_{t}(G) \leq \gamma_{t}\left(G^{\prime}\right)+1 \leq a\left(G^{\prime}\right)+2 \leq a(G)+1
$$


So, we may suppose that $d\left(v_{3}\right)=2$. Now we have three consecutive vertices $v_{1}, v_{2}, v_{3}$ with degrees $d\left(v_{1}\right)=1$ and $d\left(v_{2}\right)=d\left(v_{3}\right)=2$. Thus, by Lemma 2.1(iv), there exists a graph $G^{\prime}$ with $f\left(G^{\prime}\right)<f(G)$ which satisfies the statement.

The following lemmas will be needed to cover two specific cases in the proofs of Theorems 1.3 and 1.4. Therefore, we give the proof for both cases here.

Lemma 2.4. Let $H$ and $F \cong S\left(K_{1, \ell}\right)$ be two vertex-disjoint graphs with $n(H) \geq 3$ and $\ell \geq 2$. Assume that $w$ is a vertex of $H$ such that $H-\{w\}$ is connected and $u$ is the central vertex of the subdivided star $F$. If $G$ is the graph obtained from $H$ and $F$ by identifying $w$ and $u$, and $\gamma_{t}(G-V(F)) \leq a(G-V(F))+1$, then $\gamma_{t}(G) \leq a(G)+1$.

Proof. Suppose the subgraph $F$ of $G$ is rooted in $u$. We denote with $v_{1}, \ldots, v_{\ell}$ the children of $u$, and with $w_{1}, \ldots, w_{\ell}$ the leaves. By our assumption, $G^{\prime}=G-V(F)=G-$ $\left\{u, v_{1}, \ldots, v_{\ell}, w_{1}, \ldots, w_{\ell}\right\}$ is a nontrivial connected graph, and $m\left(G^{\prime}\right)=m(G)-d_{G}(u)-$ $\ell$. If $D^{\prime}$ is a minimum total dominating set of $G^{\prime}$, then $D=D^{\prime} \cup\left\{u, v_{1}, \ldots, v_{\ell}\right\}$ is a total dominating set of $G$, and hence $\gamma_{t}(G) \leq|D|=\left|D^{\prime}\right|+\ell+1=\gamma_{t}\left(G^{\prime}\right)+\ell+1$. Now, consider an optimal annihilation set $S^{\prime}$ in $G^{\prime}$. Independently of whether the vertices in $N_{G^{\prime}}(u)$ are inside $S^{\prime}$ or not, we have $\sum\left(S^{\prime}, G\right) \leq \sum\left(S^{\prime}, G^{\prime}\right)+d_{G}(u)-\ell \leq m\left(G^{\prime}\right)+d_{G}(u)-\ell=$ $m(G)-2 \ell$. Let $S=S^{\prime} \cup\left\{v_{1}, w_{1}, \ldots, w_{\ell}\right\}$. Then $\sum(S, G)=\sum\left(S^{\prime}, G\right)+2+\ell \leq m(G)-$ $2 \ell+\ell+2 \leq m(G)$, since $\ell \geq 2$. Then, we have $a(G) \geq|S|=\left|S^{\prime}\right|+\ell+1=a\left(G^{\prime}\right)+\ell+1$. By assumption, we have that $\gamma_{t}\left(G^{\prime}\right) \leq a\left(G^{\prime}\right)+1$. Therefore,

$$
\gamma_{t}(G) \leq \gamma_{t}\left(G^{\prime}\right)+\ell+1 \leq a\left(G^{\prime}\right)+\ell+2 \leq a(G)+1 .
$$

Lemma 2.5. Let $H$ and $P$ be two vertex-disjoint graphs, where $P$ is the paw graph and $H$ is a nontrivial connected graph. Moreover, let $z$ be a vertex of $H$ and let $x$ be a vertex of $P$ with $d_{P}(x)=2$. Assume that $G$ is the graph obtained from $H$ and $P$ by identifying $z$ and $x$. Then, there exists a connected graph $G^{\prime}$ with $f\left(G^{\prime}\right)<f(G)$ such that $\gamma_{t}\left(G^{\prime}\right) \leq a\left(G^{\prime}\right)+1$ implies $\gamma_{t}(G) \leq a(G)+1$.

Proof. If $H \cong K_{2}$, then $G$ is a graph of order 5 satisfying $\gamma_{t}(G)=2$ and $a(G)=3$. Thus, (1.1) holds for $G$. From now on, we assume that $n(H) \geq 3$. We denote the neighbors of $x$ in $P$ with $u$ and $w$, and let $v$ be the leaf neighbor of $u$. Two subcases will be considered depending on the degree $d(x)$ of $x$ in $G$.

First suppose that $d(x)=3$. Denote the third neighbor of $x$ outside $P$ with $y$. Since $H$ had at least three vertices, $y$ is not a leaf, and hence $G^{\prime}=G-V(P)=G-\{x, u, v, w\}$ is not a trivial graph. Also, $m\left(G^{\prime}\right)=m(G)-5$. If $D^{\prime}$ is a minimum total dominating set of $G^{\prime}$, then $D=D^{\prime} \cup\{u, w\}$ is a total dominating set of $G$, and hence $\gamma_{t}(G) \leq|D|=$ $\left|D^{\prime}\right|+2=\gamma_{t}\left(G^{\prime}\right)+2$. If $S^{\prime}$ is an optimal annihilation set of $G^{\prime}$, we have $\sum\left(S^{\prime}, G\right) \leq$ $\sum\left(S^{\prime}, G^{\prime}\right)+1 \leq m\left(G^{\prime}\right)+1=m(G)-4$. Let $S=S^{\prime} \cup\{u, v\}$. Then $\sum(S, G)=$ $\sum\left(S^{\prime}, G\right)+d(u)+d(v) \leq m(G)-4+3+1=m(G)$, and we have $a(G) \geq|S|=$ $\left|S^{\prime}\right|+2=a\left(G^{\prime}\right)+2$. Then, $\gamma_{t}\left(G^{\prime}\right) \leq a\left(G^{\prime}\right)+1$ implies

$$
\gamma_{t}(G) \leq \gamma_{t}\left(G^{\prime}\right)+2 \leq a\left(G^{\prime}\right)+3 \leq a(G)+1 .
$$

Now, suppose $d(x) \geq 4$. In this case let $G^{\prime}=G-\{u, v, w\}$, and so $m\left(G^{\prime}\right)=m(G)-4$. If $D^{\prime}$ is a minimum total dominating set of $G^{\prime}$, then $D=D^{\prime} \cup\{u, w\}$ is a total dominating set of graph $G$, and hence $\gamma_{t}(G) \leq|D|=\left|D^{\prime}\right|+2=\gamma_{t}\left(G^{\prime}\right)+2$. Now, let $S^{\prime}$ be an optimal annihilation set in $G^{\prime}$. If $x \notin S^{\prime}$, then $\sum\left(S^{\prime}, G\right)=\sum\left(S^{\prime}, G^{\prime}\right)$. In this case, let 
$S=S^{\prime} \cup\{u, v\}$. Then $\sum(S, G)=\sum\left(S^{\prime}, G\right)+d(u)+d(v) \leq m(G)-4+3+1=m(G)$, and we have $a(G) \geq|S|=\left|S^{\prime}\right|+2=a\left(G^{\prime}\right)+2$. If $\gamma_{t}\left(G^{\prime}\right) \leq a\left(G^{\prime}\right)+1$, the chain (2.1) of inequalities verifies the statement.

But, if $x \in S^{\prime}$, then $\sum\left(S^{\prime}, G\right)=\sum\left(S^{\prime}, G^{\prime}\right)+2 \leq m\left(G^{\prime}\right)+2=m(G)-4+$ $2=m(G)-2$. In this case, let $S=\left(S^{\prime} \backslash\{x\}\right) \cup\{u, v, w\}$. Since $d(x) \geq 4$ we have $\sum(S, G)=\sum\left(S^{\prime}, G\right)-d(x)+3+1+2 \leq m(G)-2-4+6=m(G)$, implying that $a(G) \geq|S|=\left|S^{\prime}\right|+2=a\left(G^{\prime}\right)+2$. By assumption we have that $\gamma_{t}\left(G^{\prime}\right) \leq a\left(G^{\prime}\right)+1$. Therefore, we get again (2.1) which proves the lemma.

\section{Cactus graphs}

Recall that a cactus graph is a connected graph such that any two of its cycles are pairwise edge-disjoint. If the cactus graph does not contain any cycles, then it is a tree. Let $C^{1}$ and $C^{2}$ be two cycles in the cactus graph. We define

$$
d\left(C^{1}, C^{2}\right)=\min \left\{d(u, v) \mid u \in V\left(C^{1}\right), v \in V\left(C^{2}\right)\right\}
$$

where $d(u, v)$ denotes the distance between vertices $u$ and $v$. Let $x_{1} \in V\left(C^{1}\right)$ and $x_{2} \in$ $V\left(C^{2}\right)$ be two vertices such that $d\left(x_{1}, x_{2}\right)=d\left(C^{1}, C^{2}\right)$. Then we call $x_{1}$ and $x_{2}$ exitvertices of cycles $C^{1}$ and $C^{2}$, respectively. A cycle is said to be an outer cycle if it has at most one exit-vertex. If a cactus graph is not a tree, then by the definition of a cactus graph it must contain at least one outer cycle. Note that a cactus graph, which is neither a tree nor a cycle, does not contain exit-vertices if and only if it is unicyclic. In this case, we will take an arbitrary vertex of the unique cycle whose degree is at least 3 for the role of the exit-vertex $x$. In the right-hand side graph of Figure 2, we have three possibilities for the choice of that vertex $x$ (either $x_{1}$ or $x_{2}$ or $x_{3}$ ). In both cases, whether a cactus graph has one or more cycles, vertex $x$ will always have degree $d(x) \geq 3$.
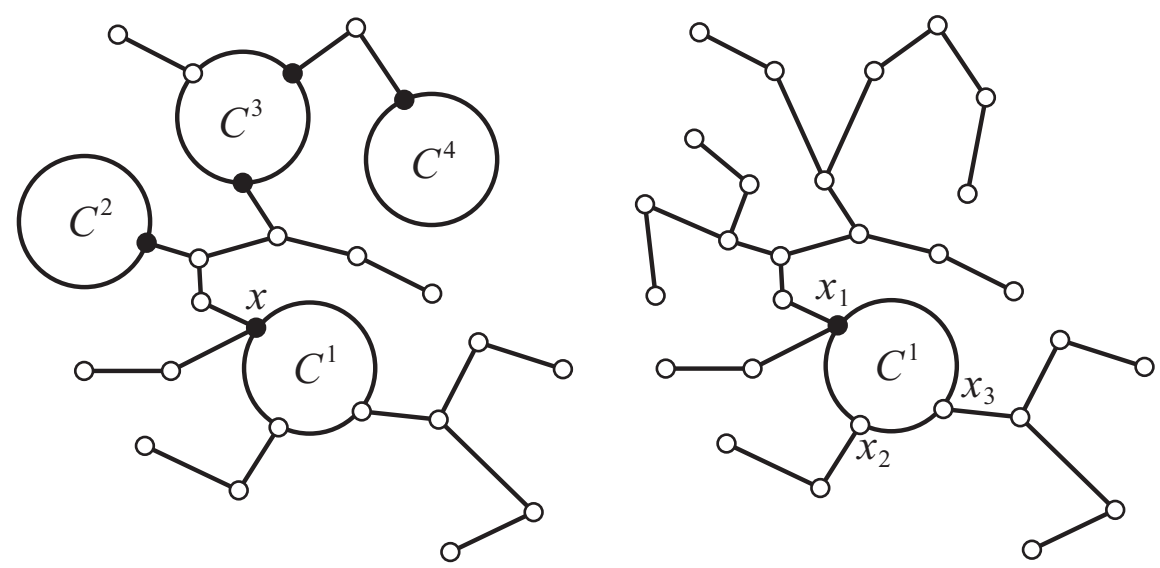

Figure 2: Two examples of cactus graphs. The first one has three outer cycles $\left(C^{1}, C^{2}, C^{4}\right)$, its exit-vertices are filled with black. The second cactus graph is unicyclic with one outer cycle, and has no exit-vertices.

In this section we prove Conjecture 1.1 for cactus graphs. Recall the corresponding statement. 
Theorem 1.3. If $G$ is a nontrivial cactus graph, then $\gamma_{t}(G) \leq a(G)+1$.

Proof. We proceed by induction on the value of function $f(G) \geq 7$. For $f(G)=7$ we have $G \cong K_{2}$, and $\gamma_{t}\left(K_{2}\right)=2=a\left(K_{2}\right)+1$. For the inductive hypothesis, let $f(G) \geq 8$ and assume that for every nontrivial cactus graph $G^{\prime}$ with $f\left(G^{\prime}\right)<f(G)$ we have $\gamma_{t}\left(G^{\prime}\right) \leq a\left(G^{\prime}\right)+1$. If $G$ is a tree, then by Theorem 1.2 the result follows. Also, if $G$ is a cycle, the statement is true. Thus, we may suppose that $G$ contains at least one cycle as a proper subgraph. We denote with $C_{k}, k \geq 3$, an outer cycle of $G$.

Through most part of the proof, we will consider cactus graphs $G^{\prime}$ formed from $G$ by removing a set of vertices in such a way that graph $G^{\prime}$ will still be a connected cactus graph and consequently $f\left(G^{\prime}\right)<f(G)$ will hold. Throughout, $S^{\prime}$ will denote an optimal annihilation set in $G^{\prime}$. We consider two cases.

Case 1: All vertices from $V\left(C_{k}\right) \backslash\{x\}$ have degree 2 .

Lemma 2.2(ii) and our inductive hypothesis together imply that $\gamma_{t}(G) \leq a(G)+1$.

Case 2: There exists a vertex from $V\left(C_{k}\right) \backslash\{x\}$ that has degree at least 3 .

Since $V\left(C_{k}\right) \backslash\{x\}$ contains some vertices of degree at least 3 , and $C_{k}$ is an outer cycle, there are trees attached to those vertices. Suppose, we root all trees in the vertices $V\left(C_{k}\right) \backslash\{x\}$ to which these trees are attached. Amongst those trees we consider the tree $T$ with the largest height $h(T)=\max \left\{d(u, v) \mid u=V\left(C_{k}\right) \cap V(T), v \in V(T)\right\}$. Denote this maximum height with $h \geq 1$ and let $u$ be the vertex of $V\left(C_{k}\right) \backslash\{x\}$ to which tree $T$ is attached. We consider three subcases.

Case 2.1: $h \geq 3$.

Since $h \geq 3$, there exists a leaf $v \in V(T)$ such that $d(u, v)=h \geq 3$. By Lemma 2.3 and our inductive hypothesis, graph $G$ satisfies (1.1).

Case 2.2: $h=2$.

We only need to consider the four cases shown in Figure 3. All other cases for $h=2$ can be proved with the help of Lemma 2.1(ii) and 2.1(v).

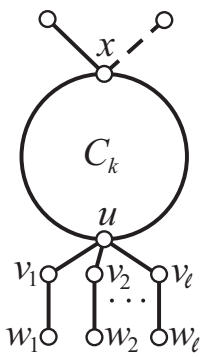

(a)

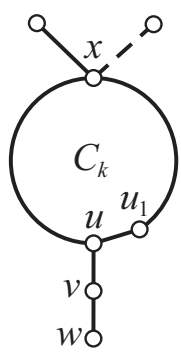

(b)

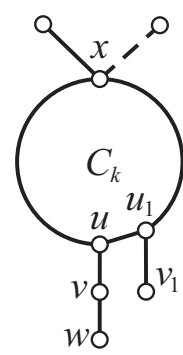

(c)

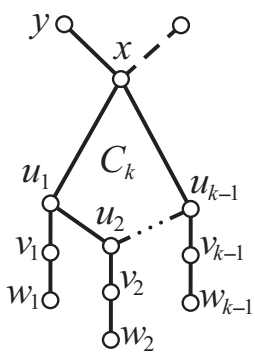

(d)

Figure 3: Cases for $h=2$.

We first start with the case in Figure 3(a). In this case, we have a subdivided star $K_{1, \ell}$, $\ell \geq 2$, attached to the outer cycle, and hence, by Lemma 2.4 and our inductive hypothesis for $G^{\prime}=G-V\left(S\left(K_{1, \ell}\right)\right)$, graph $G$ satisfies (1.1).

Next, we consider the case in Figure 3(b). Vertex $u$ has only one path of length 2 attached to it, i.e. $d(u)=3$. We suppose that $u$ has a neighbor $u_{1}$ in $V\left(C_{k}\right) \backslash\{x\}$ with 
degree $d\left(u_{1}\right)=2$. We denote with $v$ the only child of $u$, and with $w$ the only child of $v$. Let $G^{\prime}=G-\left\{u, u_{1}, v, w\right\}$, and so $m\left(G^{\prime}\right)=m(G)-5$. If $D^{\prime}$ is a minimum total dominating set of $G^{\prime}$, then $D=D^{\prime} \cup\{u, v\}$ is a total dominating set of graph $G$, and hence $\gamma_{t}(G) \leq|D|=\left|D^{\prime}\right|+2=\gamma_{t}\left(G^{\prime}\right)+2$. Independently of whether the neighbors of $u$ and $u_{1}$ in $G^{\prime}$ are inside $S^{\prime}$ or not, we have $\sum\left(S^{\prime}, G\right) \leq \sum\left(S^{\prime}, G^{\prime}\right)+2 \leq m\left(G^{\prime}\right)+2=m(G)-3$. Let $S=S^{\prime} \cup\{v, w\}$. Then $\sum(S, G)=\sum\left(S^{\prime}, G\right)+d(v)+d(w) \leq m(G)-3+2+1=$ $m(G)$, and we have $a(G) \geq|S|=\left|S^{\prime}\right|+2=a\left(G^{\prime}\right)+2$. Applying our inductive hypothesis to $G^{\prime}$, we have that $\gamma_{t}\left(G^{\prime}\right) \leq a\left(G^{\prime}\right)+1$. Therefore,

$$
\gamma_{t}(G) \leq \gamma_{t}\left(G^{\prime}\right)+2 \leq a\left(G^{\prime}\right)+3 \leq a(G)+1
$$

We proceed with the case in Figure 3(c). Vertex $u$ has again only one path of length 2 attached to it, i.e. $d(u)=3$. We suppose that $u$ has a neighbor $u_{1}$ in $V\left(C_{k}\right) \backslash\{x\}$ with degree $d\left(u_{1}\right)=3$, and a path of length 1 attached to it. Denote its child with $v_{1}$. We also denote with $v$ the only child of $u$, and with $w$ the only child of $v$. Let $G^{\prime}=$ $G-\left\{u, v, w, u_{1}, v_{1}\right\}$, and so $m\left(G^{\prime}\right)=m(G)-6$. If $D^{\prime}$ is a minimum total dominating set of $G^{\prime}$, then $D=D^{\prime} \cup\left\{u, v, u_{1}\right\}$ is a total dominating set of $G$, and hence $\gamma_{t}(G) \leq$ $|D|=\left|D^{\prime}\right|+3=\gamma_{t}\left(G^{\prime}\right)+3$. Independently of whether the neighbors of $u$ and $u_{1}$ in $G^{\prime}$ are inside $S^{\prime}$ or not, we have $\sum\left(S^{\prime}, G\right) \leq \sum\left(S^{\prime}, G^{\prime}\right)+2 \leq m\left(G^{\prime}\right)+2=m(G)-4$. Let $S=S^{\prime} \cup\left\{v, w, v_{1}\right\}$. Then $\sum(S, G)=\sum\left(S^{\prime}, G\right)+d(v)+d(w)+d\left(v_{1}\right) \leq m(G)-$ $4+2+1+1=m(G)$, and we have $a(G) \geq|S|=\left|S^{\prime}\right|+3=a\left(G^{\prime}\right)+3$. Applying our inductive hypothesis to $G^{\prime}$, we have that $\gamma_{t}\left(G^{\prime}\right) \leq a\left(G^{\prime}\right)+1$. Therefore,

$$
\gamma_{t}(G) \leq \gamma_{t}\left(G^{\prime}\right)+3 \leq a\left(G^{\prime}\right)+4 \leq a(G)+1
$$

The last case to consider is the one shown in Figure 3(d). Denote with $u_{1}, \ldots, u_{k-1}$ all vertices of $V(C) \backslash\{x\}$. Each of those vertices must have one path of length 2 attached to it, i.e. $d\left(u_{i}\right)=3$ for every $i \in\{1, \ldots, k-1\}$, since otherwise this case would be covered by one of the previous three cases. Clearly, vertices $u_{1}$ and $u_{k-1}$ are neighbors of $x$. Denote for every $i \in\{1, \ldots, k-1\}$ with $v_{i}$ the only child of $u_{i}$, and with $w_{i}$ the only child of $v_{i}$. We consider two subcases.

First, suppose that $d(x)=3$. Denote the third neighbor of $x$ outside $C_{k}$ with $y$. If vertex $y$ was a leaf, then we could exchange vertex $x$ with one of $u_{i}$ 's, and use the proof for the case in Figure 3(c). Hence, we may assume that $y$ is not a leaf and graph $G^{\prime}=$ $G-\left\{x, u_{1}, \ldots, u_{k-1}, v_{1}, \ldots, v_{k-1}, w_{1}, \ldots, w_{k-1}\right\}$ is not a trivial cactus graph. Also, $m\left(G^{\prime}\right)=m(G)-3 k+1$. If $D^{\prime}$ is a minimum total dominating set of $G^{\prime}$, then $D=$ $D^{\prime} \cup\left\{u_{1}, \ldots, u_{k-1}, v_{1}, \ldots, v_{k-1}\right\}$ is a total dominating set of $G$, and hence $\gamma_{t}(G) \leq$ $|D|=\left|D^{\prime}\right|+2 k-2=\gamma_{t}\left(G^{\prime}\right)+2 k-2$. Independently of whether $y$ is inside $S^{\prime}$ or not we have $\sum\left(S^{\prime}, G\right) \leq \sum\left(S^{\prime}, G^{\prime}\right)+1 \leq m\left(G^{\prime}\right)+1=m(G)-3 k+2$. Let $S=S^{\prime} \cup\left\{v_{1}, \ldots, v_{k-1}, w_{1}, \ldots, w_{k-1}\right\}$. Then $\sum(S, G)=\sum\left(S^{\prime}, G\right)+2(k-1)+(k-1) \leq$ $m(G)-3 k+2+(3 k-3)=m(G)-1$, and we have $a(G) \geq|S|=\left|S^{\prime}\right|+2 k-2=$ $a\left(G^{\prime}\right)+2 k-2$. Applying our inductive hypothesis to $G^{\prime}$, we have that $\gamma_{t}\left(G^{\prime}\right) \leq a\left(G^{\prime}\right)+1$. Therefore,

$$
\gamma_{t}(G) \leq \gamma_{t}\left(G^{\prime}\right)+2 k-2 \leq a\left(G^{\prime}\right)+2 k-1 \leq a(G)+1 .
$$

Now, suppose that $d(x) \geq 4$. Let $G^{\prime}=G-\left\{u_{1}, \ldots, u_{k-1}, v_{1}, \ldots, v_{k-1}, w_{1}, \ldots\right.$, $\left.w_{k-1}\right\}$, and so $m\left(G^{\prime}\right)=m(G)-3 k+2$. If $D^{\prime}$ is a minimum total dominating set of $G^{\prime}$, then $D=D^{\prime} \cup\left\{u_{1}, \ldots, u_{k-1}, v_{1}, \ldots, v_{k-1}\right\}$ is a total dominating set of $G$, and hence $\gamma_{t}(G) \leq|D|=\left|D^{\prime}\right|+2 k-2=\gamma_{t}\left(G^{\prime}\right)+2 k-2$. If $x \notin S^{\prime}$, then $\sum\left(S^{\prime}, G\right)=\sum\left(S^{\prime}, G^{\prime}\right)$. 
In this case, let $S=S^{\prime} \cup\left\{v_{1}, \ldots, v_{k-1}, w_{1}, \ldots, w_{k-1}\right\}$. Then $\sum(S, G)=\sum\left(S^{\prime}, G\right)+$ $2(k-1)+(k-1) \leq m(G)-1$, and we have $a(G) \geq|S|=\left|S^{\prime}\right|+2 k-2=a\left(G^{\prime}\right)+2 k-2$. Applying our inductive hypothesis to $G^{\prime}$, we have that $\gamma_{t}\left(G^{\prime}\right) \leq a\left(G^{\prime}\right)+1$. Therefore,

$$
\gamma_{t}(G) \leq \gamma_{t}\left(G^{\prime}\right)+2 k-2 \leq a\left(G^{\prime}\right)+2 k-1 \leq a(G)+1 .
$$

If $x \in S^{\prime}$, then $\sum\left(S^{\prime}, G\right)=\sum\left(S^{\prime}, G^{\prime}\right)+2 \leq m\left(G^{\prime}\right)+2=m(G)-3 k+2+2=$ $m(G)-3 k+4$. In this case, let $S=\left(S^{\prime} \backslash\{x\}\right) \cup\left\{u_{1}, v_{1}, \ldots, v_{k-1}, w_{1}, \ldots, w_{k-1}\right\}$. Since $d(x) \geq 4$ we have $\sum(S, G)=\sum\left(S^{\prime}, G\right)-d(x)+d\left(u_{1}\right)+2(k-1)+(k-1) \leq m(G)-3 k+$ $4-4+3+3(k-1)=m(G)$, implying that $a(G) \geq|S|=\left|S^{\prime}\right|+2 k-2=a\left(G^{\prime}\right)+2 k-2$. By our inductive hypothesis, we have that $\gamma_{t}\left(G^{\prime}\right) \leq a\left(G^{\prime}\right)+1$. Therefore,

$$
\gamma_{t}(G) \leq \gamma_{t}\left(G^{\prime}\right)+2 k-2 \leq a\left(G^{\prime}\right)+2 k-1 \leq a(G)+1 .
$$

Case 2.3: $h=1$.

It suffices to consider only those cases shown in Figure 4. Note that all other cactus graphs with $h=1$ would involve two leaves at distance of at most 3, and hence these cases can be reduced to the direct application of Lemma 2.1(ii) and 2.1(v).

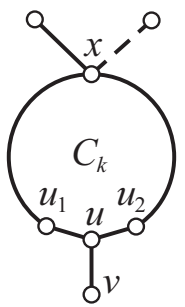

(a)

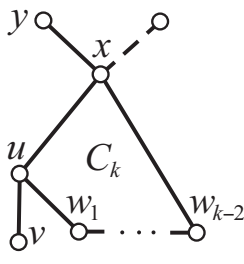

(b)

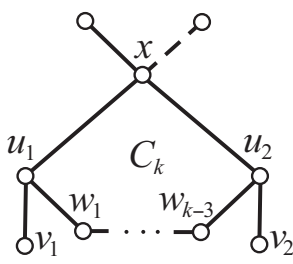

(c)

Figure 4: Cases for $h=1$.

First, consider Figure 4(a). Here, we assume that vertex $u$ has degree $d(u)=3$, and its neighbors in $V\left(C_{k}\right) \backslash\{x\}$, namely $u_{1}$ and $u_{2}$, are of degree 2 . Denote the child of $u$ with $v$. In this case we want $u_{1}$ and $u_{2}$ to be different from the exit-vertex $x$. Let $G^{\prime}=$ $G-\left\{u, v, u_{1}, u_{2}\right\}$, and so $m\left(G^{\prime}\right)=m(G)-5$. If $D^{\prime}$ is a minimum total dominating set of $G^{\prime}$, then $D=D^{\prime} \cup\left\{u, u_{i}\right\}$ with $i=1$ or $i=2$ is a total dominating set of $G$, and hence $\gamma_{t}(G) \leq|D|=\left|D^{\prime}\right|+2=\gamma_{t}\left(G^{\prime}\right)+2$. Independently of whether the neighbors of $u_{1}$ and $u_{2}$ in $G^{\prime}$ are inside $S^{\prime}$ or not, we have $\sum\left(S^{\prime}, G\right) \leq \sum\left(S^{\prime}, G^{\prime}\right)+2 \leq m\left(G^{\prime}\right)+2=m(G)-3$. Let $S=S^{\prime} \cup\left\{u_{1}, v\right\}$. Then $\sum(S, G)=\sum\left(S^{\prime}, G\right)+d\left(u_{1}\right)+d(v) \leq m(G)-3+2+1=$ $m(G)$, and we have $a(G) \geq|S|=\left|S^{\prime}\right|+2=a\left(G^{\prime}\right)+2$. Applying our inductive hypothesis to $G^{\prime}$, we have that $\gamma_{t}\left(G^{\prime}\right) \leq a\left(G^{\prime}\right)+1$. Therefore,

$$
\gamma_{t}(G) \leq \gamma_{t}\left(G^{\prime}\right)+2 \leq a\left(G^{\prime}\right)+3 \leq a(G)+1 .
$$

We proceed with the case in Figure 4(b). Denote with $u$ the vertex of $V\left(C_{k}\right) \backslash\{x\}$ with one path of length 1 attached to it, i.e. $d(u)=3$, and let $v$ be its only child. One of the neighbors of $u$ must clearly be vertex $x$ because otherwise we would have the case in Figure 4(a). Suppose that all other vertices in $V\left(C_{k}\right) \backslash\{x\}$, denote them with $w_{1}, \ldots, w_{k-2}$, have degree 2 . 
First suppose that $k=3$. In this case $x, u, v$ and $w_{1}$ induce the paw graph. Then, by Lemma 2.5 and our inductive hypothesis, graph $G$ satisfies (1.1).

Suppose that $k \geq 4$. Let $G^{\prime}=G-\left\{u, v, w_{1}, w_{2}\right\}$, and so $m\left(G^{\prime}\right)=m(G)-5$. Remark that $G^{\prime}$ remains a cactus graph. If $D^{\prime}$ is a minimum total dominating set of $G^{\prime}$, then $D=D^{\prime} \cup\left\{u, w_{1}\right\}$ is a total dominating set of $G$, and hence $\gamma_{t}(G) \leq|D|=\left|D^{\prime}\right|+2=$ $\gamma_{t}\left(G^{\prime}\right)+2$. Independently of whether $x$ and $w_{3}$ is inside $S^{\prime}$ or not we have $\sum\left(S^{\prime}, G\right) \leq$ $\sum\left(S^{\prime}, G^{\prime}\right)+2 \leq m\left(G^{\prime}\right)+2=m(G)-3$. Let $S=S^{\prime} \cup\left\{v, w_{1}\right\}$. Then $\sum(S, G)=$ $\sum\left(S^{\prime}, G\right)+d(v)+d\left(w_{1}\right) \leq m(G)-3+1+2=m(G)$, and we have $a(G) \geq|S|=\left|S^{\prime}\right|+$ $2=a\left(G^{\prime}\right)+2$. Applying our inductive hypothesis to $G^{\prime}$, we have that $\gamma_{t}\left(G^{\prime}\right) \leq a\left(G^{\prime}\right)+1$. Therefore,

$$
\gamma_{t}(G) \leq \gamma_{t}\left(G^{\prime}\right)+2 \leq a\left(G^{\prime}\right)+3 \leq a(G)+1
$$

We finish with the case in Figure 4(c). Denote with $u_{1}$ and $u_{2}$ two vertices in $V\left(C_{k}\right) \backslash\{x\}$ each with one path of length 1 attached to it, i.e. $d\left(u_{1}\right)=d\left(u_{2}\right)=3$, and let $v_{1}$ and $v_{2}$ be the only child of $u_{1}$ and $u_{2}$, respectively. The exit-vertex $x$ must be the neighbor of both $u_{1}$ and $u_{2}$ because otherwise we would have the case in Figure 4(a). We denote all vertices in $V\left(C_{k}\right) \backslash\{x\}$ between vertex $u_{1}$ and $u_{2}$ with $w_{1}, \ldots, w_{k-3}$. Those vertices have all degree 2 .

If $k=3$, the statement follows immediately from the hypothesis and Lemma 2.1(v), since in this case the support vertices of $v_{1}$ and $v_{2}$ are adjacent. Thus, we first suppose that $k=4$. Let $G^{\prime}=G-\left\{u_{1}, v_{1}, u_{2}, v_{2}, w_{1}\right\}$, and so $m\left(G^{\prime}\right)=m(G)-6$. If $D^{\prime}$ is a minimum total dominating set of $G^{\prime}$, then $D=D^{\prime} \cup\left\{x, u_{1}, u_{2}\right\}$ is a total dominating set of $G$, and hence $\gamma_{t}(G) \leq|D|=\left|D^{\prime}\right|+3=\gamma_{t}\left(G^{\prime}\right)+3$. Independently of whether $x \in S^{\prime}$ or $x \notin S^{\prime}$, we have $\sum\left(S^{\prime}, G\right) \leq \sum\left(S^{\prime}, G^{\prime}\right)+2 \leq m\left(G^{\prime}\right)+2=m(G)-4$. Let $S=S^{\prime} \cup\left\{v_{1}, v_{2}, w_{1}\right\}$. Then $\sum(S, G)=\sum\left(S^{\prime}, G\right)+d\left(v_{1}\right)+d\left(v_{2}\right)+d\left(w_{1}\right) \leq m(G)-4+1+1+2=m(G)$, and we have $a(G) \geq|S|=\left|S^{\prime}\right|+3=a\left(G^{\prime}\right)+3$. Applying our inductive hypothesis to $G^{\prime}$, we have that $\gamma_{t}\left(G^{\prime}\right) \leq a\left(G^{\prime}\right)+1$. Therefore,

$$
\gamma_{t}(G) \leq \gamma_{t}\left(G^{\prime}\right)+3 \leq a\left(G^{\prime}\right)+4 \leq a(G)+1
$$

Now, suppose that $k=5$. We make a similar cut than the one for $k=4$. Let $G^{\prime}=G-\left\{u_{1}, v_{1}, u_{2}, v_{2}, w_{1}, w_{2}\right\}$, and so $m\left(G^{\prime}\right)=m(G)-7$. If $D^{\prime}$ is a minimum total dominating set of $G^{\prime}$, then $D=D^{\prime} \cup\left\{x, u_{1}, u_{2}\right\}$ is a total dominating set of $G$, and hence $\gamma_{t}(G) \leq|D|=\left|D^{\prime}\right|+3=\gamma_{t}\left(G^{\prime}\right)+3$. For any optimal annihilation set $S^{\prime}$ of $G^{\prime}$, we have $\sum\left(S^{\prime}, G\right) \leq \sum\left(S^{\prime}, G^{\prime}\right)+2 \leq m\left(G^{\prime}\right)+2=m(G)-5$. Let $S=S^{\prime} \cup\left\{v_{1}, v_{2}, w_{1}\right\}$. Then $\sum(S, G)=\sum\left(S^{\prime}, G\right)+d\left(v_{1}\right)+d\left(v_{2}\right)+d\left(w_{1}\right) \leq m(G)-1$, and $a(G) \geq|S|=\left|S^{\prime}\right|+3=a\left(G^{\prime}\right)+3$ follows. Applying our inductive hypothesis to $G^{\prime}$, we have that $\gamma_{t}\left(G^{\prime}\right) \leq a\left(G^{\prime}\right)+1$. Therefore,

$$
\gamma_{t}(G) \leq \gamma_{t}\left(G^{\prime}\right)+3 \leq a\left(G^{\prime}\right)+4 \leq a(G)+1
$$

For the last case, let $k \geq 6$. We have three consecutive vertices $w_{1}, w_{2}, w_{3}$ with degree $d\left(w_{1}\right)=d\left(w_{2}\right)=d\left(w_{3}\right)=2$. Furthermore, vertices $u_{1}$ and $w_{4}$ (or $u_{1}$ and $u_{2}$, if $k=6$ ) are not adjacent. Thus, by Lemma 2.1(iii) and our inductive hypothesis, graph $G$ satisfies (1.1).

These cover all possible cases which can occur in a cactus graph which is neither a tree nor a cycle. Hence, Conjecture 1.1 is true for the family of cactus graphs. 


\section{Block graphs}

Recall that a block graph is a connected graph in which every 2-connected component (block) is a clique. Block graphs have minimum degree at least 3 if its building blocks are complete graphs $K_{k}, k \geq 4$. Thus, Conjecture 1.1 obviously holds for them. On the other hand, block graphs also contain blocks $K_{2}$ and $K_{3}$, and therefore, it clearly makes sense to study Conjecture 1.1 on block graphs.

We proceed with a similar definition than the one for cactus graphs. If all cliques in a block graph are $K_{2}$, then it is a tree. For every $k \geq 3$ we will call complete graph $K_{k}$ a complex clique. Let $K^{1}$ and $K^{2}$ be two complex cliques in the block graph. We define

$$
d\left(K^{1}, K^{2}\right)=\min \left\{d(u, v) \mid u \in V\left(K^{1}\right), v \in V\left(K^{2}\right)\right\},
$$

where $d(u, v)$ denotes the distance between vertices $u$ and $v$. Let $x_{1} \in V\left(K^{1}\right)$ and $x_{2} \in$ $V\left(K^{2}\right)$ be two vertices such that $d\left(x_{1}, x_{2}\right)=d\left(K^{1}, K^{2}\right)$. Then we call $x_{1}$ and $x_{2}$ exitvertices of complex cliques $K^{1}$ and $K^{2}$, respectively. Notice that a complex clique might not have any exit-vertices if it is the only complex clique in the block graph. A complex clique will be called an outer complex clique if it has at most one exit-vertex. If a block graph is not a tree, then by the definition of a block graph it must contain at least one outer complex clique.

Now, we are ready to present a proof of Theorem 1.4. Recall its statement.

Theorem 1.4. If $G$ is a nontrivial block graph, then $\gamma_{t}(G) \leq a(G)+1$.

Proof. We proceed by induction on the value of function $f(G)$. For $f(G)=7$ we have $G \cong K_{2}$, and $\gamma_{t}\left(K_{2}\right)=2=a\left(K_{2}\right)+1$. For the inductive hypothesis, let $f(G) \geq 8$ and assume that for every nontrivial block graph $G^{\prime}$ with $f\left(G^{\prime}\right)<f(G)$ we have $\gamma_{t}\left(G^{\prime}\right) \leq$ $a\left(G^{\prime}\right)+1$. If $G$ does not contain complex cliques, then it is a tree, and by Theorem 1.2 the result follows. Also, if $G$ is a complete graph, i.e. $G \cong K_{\ell}, \ell \geq 2$, we have $\gamma_{t}\left(K_{\ell}\right)=$ $2 \leq a\left(K_{\ell}\right)+1$. Thus, we may suppose that $G$ is neither a tree nor a complete graph, but contains at least one complex clique as a proper subgraph. We denote with $K_{k}$ an outer complex clique of $G$. Similarly as in the proof for cactus graphs, all outer complex cliques in the figures will be drawn with an exit-vertex $x$ even though a unique complex clique in a block graph does not have one. In the latter case, we denote with $x$ an arbitrary vertex of clique $K_{k}$ whose degree is at least $k$. In both cases, whether a block graph has one or more complex cliques, vertex $x$ will have degree $d(x) \geq k$.

Through most part of the proof, we will consider block graphs $G^{\prime}$ formed from $G$ by removing a set of vertices in such a way that graph $G^{\prime}$ will still be a connected block graph and consequently $f\left(G^{\prime}\right)<f(G)$ will hold. Throughout, $S^{\prime}$ will denote an optimal annihilation set in $G^{\prime}$. We consider two cases.

Case 1: All vertices from $V\left(K_{k}\right) \backslash\{x\}$ have degree $k-1$.

Let $u_{1}, \ldots, u_{k-1}$ be vertices from $V\left(K_{k}\right) \backslash\{x\}$ with degree $k-1$. By Lemma 2.2(i), and inductive hypothesis for $G^{\prime}=G-\left\{u_{1}, \ldots, u_{k-1}\right\}$, graph $G$ satisfies (1.1).

Case 2: There exists a vertex from $V\left(K_{k}\right) \backslash\{x\}$ that has degree at least $k$.

Since $V\left(K_{k}\right) \backslash\{x\}$ contains vertices of degree at least $k$, and $K_{k}$ is an outer complex clique, there are trees attached to those vertices. Suppose, we root all trees in the vertices $V\left(K_{k}\right) \backslash\{x\}$ to which these trees are attached. Amongst those trees we consider the tree 
$T$ with the largest height $h$. Let $u$ be the vertex of $V\left(K_{k}\right) \backslash\{x\}$ to which this tree $T$ is attached. We split the problem into three subcases.

Case 2.1: $h \geq 3$.

Since $h \geq 3$, there exists a leaf $v \in V(T)$ such that $d(u, v)=d \geq 3$. By Lemma 2.3 and our inductive hypothesis, graph $G$ satisfies (1.1).

Case 2.2: $h=2$.

We only need to consider cases shown in Figure 5. All other cases for $h=2$ can be proved directly with the help of Lemma 2.1(ii) and 2.1(v).

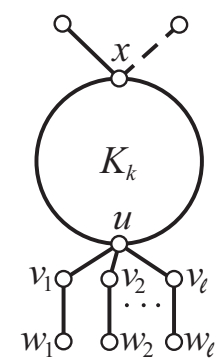

(a)

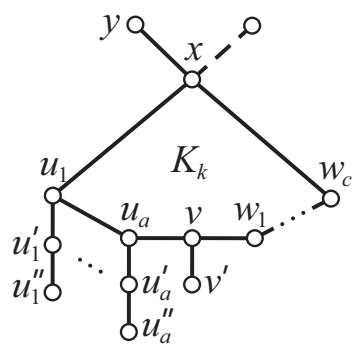

(b)

Figure 5: Cases for $h=2$.

We start with the case in Figure 5(a) and suppose that there exists a subdivided star $S\left(K_{1, \ell}\right), \ell \geq 2$, attached to the outer complex clique. By Lemma 2.4 and our inductive hypothesis for $G^{\prime}=G-V\left(S\left(K_{1, \ell}\right)\right)$, graph $G$ satisfies (1.1).

In the case shown in Figure 5(b), there are vertices in $V\left(K_{k}\right) \backslash\{x\}$ such that a path of length 2 is attached to them. We denote such vertices with $u_{1}, \ldots, u_{a}$. Since $h=2$, we must have at least one such vertex. Thus, $a \in\{1, \ldots, k-1\}$. For each $i \in\{1, \ldots, a\}$ we denote with $u_{i}^{\prime}$ the child of $u_{i}$, and with $u_{i}^{\prime \prime}$ the child of $u_{i}^{\prime}$. Also, we may suppose that at most one vertex in $V\left(K_{k}\right) \backslash\{x\}$ has a path of length 1 attached to it. If we had more such vertices, then we would have two adjacent support vertices and we could prove the statement by referring to Lemma 2.1(v). Hence, denote this vertex with $v$ and let $b$ denote the Boolean value whether it exists in $V\left(K_{k}\right) \backslash\{x\}$ or not, i.e. $b \in\{0,1\}$. We denote the child of $v$ with $v^{\prime}$. There may also be some vertices in $V\left(K_{k}\right) \backslash\{x\}$ without a path attached to them. Denote them with $w_{1}, \ldots, w_{c}, c \in\{0, \ldots, k-2\}$. Clearly, we have $a+b+c=k-1$. Let $G^{\prime}=G-\left\{u_{1}, \ldots u_{a}, u_{1}^{\prime}, \ldots u_{a}^{\prime}, u_{1}^{\prime \prime}, \ldots, u_{a}^{\prime \prime}, v, v^{\prime}, w_{1}, \ldots, w_{c}\right\}$, and so $m\left(G^{\prime}\right)=m(G)-\left(\frac{k(k-1)}{2}+2 a+b\right)$. If $D^{\prime}$ is a minimum total dominating set of $G^{\prime}$, then $D=D^{\prime} \cup\left\{u_{1}, \ldots, u_{a}, u_{1}^{\prime}, \ldots, u_{a}^{\prime}, v\right\}$ is a total dominating set of $G$, and hence $\gamma_{t}(G) \leq|D|=\left|D^{\prime}\right|+2 a+b=\gamma_{t}\left(G^{\prime}\right)+2 a+b$. Independently of whether $x$ is inside $S^{\prime}$ or not we have

$$
\begin{aligned}
\sum\left(S^{\prime}, G\right) & \leq \sum\left(S^{\prime}, G^{\prime}\right)+(k-1) \\
& =m\left(G^{\prime}\right)+(k-1)=m(G)-\left(\frac{k(k-1)}{2}+2 a+b\right)+k-1
\end{aligned}
$$


Let $S=S^{\prime} \cup\left\{u_{1}^{\prime}, \ldots, u_{a}^{\prime}, u_{1}^{\prime \prime}, \ldots u_{a}^{\prime \prime}, v^{\prime}\right\}$ and observe that

$$
\begin{aligned}
\sum(S, G) & =\sum\left(S^{\prime}, G\right)+d\left(u_{1}^{\prime}\right)+\cdots+d\left(u_{a}^{\prime}\right)+d\left(u_{1}^{\prime \prime}\right)+\cdots+d\left(u_{a}^{\prime \prime}\right)+d\left(v^{\prime}\right) \\
& \leq m(G)-\left(\frac{k(k-1)}{2}+2 a+b\right)+k-1+3 a+b .
\end{aligned}
$$

First, suppose that $1 \leq a \leq k-2$ holds. Then,

$$
\begin{array}{r}
m(G)-\left(\frac{k(k-1)}{2}+2 a+b\right)+k-1+3 a+b=m(G)-\frac{1}{2} k^{2}+\frac{3}{2} k+a-1 \\
\leq m(G)-\frac{1}{2} k^{2}+\frac{5}{2} k-3=m(G)-\frac{1}{2}(k-2)(k-3) \leq m(G) .
\end{array}
$$

Similarly, under the conditions $a=k-1 \geq 3$, the following relations hold:

$$
m(G)-\left(\frac{k(k-1)}{2}+2 a+b\right)+k-1+3 a+b \leq m(G)-\frac{1}{2} k^{2}+\frac{5}{2} k-2 \leq m(G) .
$$

In both cases we get $\sum(S, G) \leq m(G)$, which implies $a(G) \geq|S|=\left|S^{\prime}\right|+2 a+b=$ $a\left(G^{\prime}\right)+2 a+b$. By our inductive hypothesis, $G^{\prime}$ satisfies Conjecture 1.1. Consequently,

$$
\gamma_{t}(G) \leq \gamma_{t}\left(G^{\prime}\right)+2 a+b \leq a\left(G^{\prime}\right)+2 a+b+1 \leq a(G)+1 .
$$

What remains is to establish the statement for $k=3$ and $a=k-1=2$. We consider two subcases. First, suppose that $d(x)=3$. Denote the third neighbor of $x$ outside $K_{3}$ with $y$. If vertex $y$ was a leaf, then we could exchange vertex $x$ either with $u_{1}$ or $u_{2}$, and apply the proof for the case $a=1=k-2$. Hence, we may assume that $y$ is not a leaf, and therefore, graph $G^{\prime}=G-\left\{x, u_{1}, u_{1}^{\prime}, u_{1}^{\prime \prime}, u_{2}, u_{2}^{\prime}, u_{2}^{\prime \prime}\right\}$ is not a trivial block graph. Also, $m\left(G^{\prime}\right)=m(G)-8$. If $D^{\prime}$ is a minimum total dominating set of $G^{\prime}$, then $D=D^{\prime} \cup\left\{u_{1}, u_{1}^{\prime}, u_{2}, u_{2}^{\prime}\right\}$ is a total dominating set of graph $G$, and hence $\gamma_{t}(G) \leq|D|=$ $\left|D^{\prime}\right|+4=\gamma_{t}\left(G^{\prime}\right)+4$. Independently of whether $y$ is inside $S^{\prime}$ or not we have $\sum\left(S^{\prime}, G\right) \leq$ $\sum\left(S^{\prime}, G^{\prime}\right)+1 \leq m\left(G^{\prime}\right)+1=m(G)-8+1=m(G)-7$. Let $S=S^{\prime} \cup\left\{u_{1}^{\prime}, u_{1}^{\prime \prime}, u_{2}^{\prime}, u_{2}^{\prime \prime}\right\}$. Then $\sum(S, G)=\sum\left(S^{\prime}, G\right)+d\left(u_{1}^{\prime}\right)+d\left(u_{\ell}^{\prime \prime}\right)+d\left(u_{2}^{\prime}\right)+d\left(u_{2}^{\prime \prime}\right) \leq m(G)-7+2+1+2+1=$ $m(G)-1$, and we have $a(G) \geq|S|=\left|S^{\prime}\right|+4=a\left(G^{\prime}\right)+4$. Applying our inductive hypothesis to $G^{\prime}$, we have that $\gamma_{t}\left(G^{\prime}\right) \leq a\left(G^{\prime}\right)+1$. Therefore,

$$
\gamma_{t}(G) \leq \gamma_{t}\left(G^{\prime}\right)+4 \leq a\left(G^{\prime}\right)+5 \leq a(G)+1 .
$$

Now, suppose that $d(x) \geq 4$. Let $G^{\prime}=G-\left\{u_{1}, u_{1}^{\prime}, u_{1}^{\prime \prime}, u_{2}, u_{2}^{\prime}, u_{2}^{\prime \prime}\right\}$, and so $m\left(G^{\prime}\right)=$ $m(G)-7$. If $D^{\prime}$ is a minimum total dominating set of $G^{\prime}$, then $D=D^{\prime} \cup\left\{u_{1}, u_{1}^{\prime}, u_{2}, u_{2}^{\prime}\right\}$ is a total dominating set of $G$, and hence $\gamma_{t}(G) \leq|D|=\left|D^{\prime}\right|+4=\gamma_{t}(G)+4$. If $x \notin S^{\prime}$, then $\sum\left(S^{\prime}, G\right)=\sum\left(S^{\prime}, G^{\prime}\right)$. In this case, let $S=S^{\prime} \cup\left\{u_{1}^{\prime}, u_{1}^{\prime \prime}, u_{2}^{\prime}, u_{2}^{\prime \prime}\right\}$. Then $\sum(S, G)=$ $\sum\left(S^{\prime}, G\right)+d\left(u_{1}^{\prime}\right)+d\left(u_{1}^{\prime \prime}\right)+d\left(u_{2}^{\prime}\right)+d\left(u_{2}^{\prime \prime}\right) \leq m(G)-7+2+1+2+1=m(G)-1$, and we have $a(G) \geq|S|=\left|S^{\prime}\right|+4=a\left(G^{\prime}\right)+4$. Applying our inductive hypothesis to $G^{\prime}$, we have that $\gamma_{t}\left(G^{\prime}\right) \leq a\left(G^{\prime}\right)+1$. Therefore,

$$
\gamma_{t}(G) \leq \gamma_{t}\left(G^{\prime}\right)+4 \leq a\left(G^{\prime}\right)+5 \leq a(G)+1 .
$$

If $x \in S^{\prime}$, then $\sum\left(S^{\prime}, G\right)=\sum\left(S^{\prime}, G^{\prime}\right)+2 \leq m\left(G^{\prime}\right)+2=m(G)-7+2=m(G)-5$. In this case, let $S=\left(S^{\prime} \backslash\{x\}\right) \cup\left\{u_{1}, u_{1}^{\prime}, u_{1}^{\prime \prime}, u_{2}^{\prime}, u_{2}^{\prime \prime}\right\}$. Since $d(x) \geq 4$ we have $\sum(S, G)=$ 
$\sum\left(S^{\prime}, G\right)-d(x)+d\left(u_{1}\right)+d\left(u_{1}^{\prime}\right)+d\left(u_{1}^{\prime \prime}\right)+d\left(u_{2}^{\prime}\right)+d\left(u_{2}^{\prime \prime}\right) \leq m(G)-5-4+3+2+1+2+1=$ $m(G)$, implying that $a(G) \geq|S|=\left|S^{\prime}\right|+4=a\left(G^{\prime}\right)+4$. Applying again our inductive hypothesis to $G^{\prime}$, we have that $\gamma_{t}\left(G^{\prime}\right) \leq a\left(G^{\prime}\right)+1$. Therefore,

$$
\gamma_{t}(G) \leq \gamma_{t}\left(G^{\prime}\right)+4 \leq a\left(G^{\prime}\right)+5 \leq a(G)+1 .
$$

Case 2.3: $h=1$.

We need to consider only one case which is shown in Figure 6. As we have already seen in Case 2.2, all other cases for $h=1$ can be proved with the help of Lemma 2.1(ii) and 2.1(v).

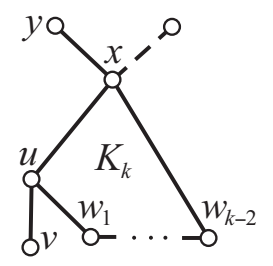

Figure 6: The case for $h=1$.

We may also suppose that there is at most one vertex in $V\left(K_{k}\right) \backslash\{x\}$ which has a path of length 1 attached to it. If we had more such vertices, then we would have adjacent support vertices and we could prove this case with Lemma 2.1(v). Denote this vertex with $u$ and its child with $v$. There are also vertices in $V\left(K_{k}\right) \backslash\{x\}$ without a path attached to them. Denote them with $w_{1}, \ldots, w_{k-2}$. Let $G^{\prime}=G-\left\{u, v, w_{1}, \ldots, w_{k-2}\right\}$, and so $m\left(G^{\prime}\right)=m(G)-\left(\frac{k(k-1)}{2}+1\right)$. If $D^{\prime}$ is a minimum total dominating set of $G^{\prime}$, then $D=$ $D^{\prime} \cup\{x, u\}$ is a total dominating set of $G$, and hence $\gamma_{t}(G) \leq|D|=\left|D^{\prime}\right|+2=\gamma_{t}\left(G^{\prime}\right)+2$. Independently of whether $x$ is inside $S^{\prime}$ or not we have $\sum\left(S^{\prime}, G\right) \leq \sum\left(S^{\prime}, G^{\prime}\right)+k-1 \leq$ $m\left(G^{\prime}\right)+k-1=m(G)-\left(\frac{k(k-1)}{2}+1\right)+k-1$. Let $S=S^{\prime} \cup\left\{v, w_{1}\right\}$. Then,

$$
\begin{aligned}
\sum(S, G) & =\sum\left(S^{\prime}, G\right)+d(v)+d\left(w_{1}\right) \\
& \leq m(G)-\left(\frac{k(k-1)}{2}+1\right)+k-1+1+k-1 .
\end{aligned}
$$

For $k \geq 4$, this gives

$$
\sum(S, G) \leq m(G)-\frac{1}{2} k^{2}+\frac{5}{2} k-2 \leq m(G) .
$$

Hence, $a(G) \geq|S|=\left|S^{\prime}\right|+2=a\left(G^{\prime}\right)+2$ and applying our inductive hypothesis to $G^{\prime}$, we have that $\gamma_{t}\left(G^{\prime}\right) \leq a\left(G^{\prime}\right)+1$. Therefore,

$$
\gamma_{t}(G) \leq \gamma_{t}\left(G^{\prime}\right)+2 \leq a\left(G^{\prime}\right)+3 \leq a(G)+1 .
$$

We end the proof with $k=3$. In this case, $x, u, v$ and $w_{1}$ induce the paw graph and, by Lemma 2.5 and our inductive hypothesis, graph $G$ satisfies (1.1).

We have considered all possible cases which can occur in a block graph which is neither a tree nor a complete graph. Hence, Conjecture 1.1 is true over the family of block graphs. 


\section{Concluding remarks}

To show that our main results, namely Theorem 1.3 and 1.4, are sharp, we remark that trees are included in both classes. Therefore, we may refer to the family of trees characterized in [5] which satisfy Conjecture 1.1 with equality.

We may also observe that even cycles $C_{n}$, where $n \equiv 2(\bmod 4)$, have $\gamma_{t}\left(C_{n}\right)=\frac{n}{2}+1$ and $a\left(C_{n}\right)=\frac{n}{2}$. Also, there are other cactus graphs which are neither trees nor cycles, but satisfy $\gamma_{t}(G)=a(G)+1$. Take two vertex-disjoint cycles $C_{6}$, and connect any vertex from the first cycle and any vertex from the second cycle with a path of length 3 . We get a cactus graph $G$ on $n=14$ vertices and $m=15$ edges. It is easy to see that $\gamma_{t}(G)=8$ and $a(G)=7$. Thus, Theorem 1.3 holds with equality for the graph $G$ constructed this way. One can also use other cycles $C_{n}$ with $n \equiv 2(\bmod 4)$ and connect them with different paths to obtain other extremal examples. Hence, the following characterization problem remains open.

Problem 5.1. Characterize cactus graphs $G$ which satisfy $\gamma_{t}(G)=a(G)+1$.

For block graphs, already the following question might be interesting.

Problem 5.2. Does there exist a block graph $G$ which is neither a tree nor a $K_{3}$ but satisfies $\gamma_{t}(G)=a(G)+1$ ?

\section{References}

[1] D. Archdeacon, J. Ellis-Monaghan, D. Fisher, D. Froncek, P. C. B. Lam, S. Seager, B. Wei and R. Yuster, Some remarks on domination, J. Graph Theory 46 (2004), 207-210, doi:10.1002/ jgt.20000.

[2] B. Brimkov and I. V. Hicks, Memory efficient algorithms for cactus graphs and block graphs, Discrete Appl. Math. 216 (2017), 393-407, doi:10.1016/j.dam.2015.10.032.

[3] V. Chvátal and C. McDiarmid, Small transversals in hypergraphs, Combinatorica 12 (1992), 19-26, doi:10.1007/bf01191201.

[4] E. DeLaViña, Written on the Wall II, http://cms.dt.uh.edu/faculty/ delavinae/research/wowII/.

[5] W. J. Desormeaux, T. W. Haynes and M. A. Henning, Relating the annihilation number and the total domination number of a tree, Discrete Appl. Math. 161 (2013), 349-354, doi:10.1016/j. dam.2012.09.006.

[6] W. J. Desormeaux, M. A. Henning, D. F. Rall and A. Yeo, Relating the annihilation number and the 2-domination number of a tree, Discrete Math. 319 (2014), 15-23, doi:10.1016/j.disc. 2013.11.020.

[7] J. R. Griggs and D. J. Kleitman, Independence and the Havel-Hakimi residue, Discrete Math. 127 (1994), 209-212, doi:10.1016/0012-365x(92)00479-b.

[8] M. A. Henning, A survey of selected recent results on total domination in graphs, Discrete Math. 309 (2009), 32-63, doi:10.1016/j.disc.2007.12.044.

[9] M. A. Henning and A. Yeo, Total Domination in Graphs, Springer Monographs in Mathematics, Springer, New York, 2013, doi:10.1007/978-1-4614-6525-6.

[10] J. Lyle and S. Patterson, A note on the annihilation number and 2-domination number of a tree, J. Comb. Optim. 33 (2017), 968-976, doi:10.1007/s10878-016-0019-7. 
[11] R. Pepper, On the annihilation number of a graph, in: V. Zafiris, M. Benavides, K. Gao, S. Hashemi, K. Jegdic, G. A. Kouzaev, P. Simeonov, L. Vladareanu and C. Vobach (eds.), AMATH'09 Proceedings of the 15th American Conference on Applied Mathematics, World Scientific and Engineering Academy and Society (WSEAS), Stevens Point, Wisconsin, USA, 2009 pp. 217-220, held in Houston, USA, April 30 - May 02, 2009.

[12] R. D. Pepper, Binding Independence, Ph.D. thesis, University of Houston, Houston, Texas, 2004, https://search.proquest.com/docview/305196562.

[13] S. Thomassé and A. Yeo, Total domination of graphs and small transversals of hypergraphs, Combinatorica 27 (2007), 473-487, doi:10.1007/s00493-007-2020-3.

[14] Zs. Tuza, Covering all cliques of a graph, Discrete Math. 86 (1990), 117-126, doi:10.1016/ 0012-365x(90)90354-k. 